\title{
BMJ Open Surgery for Type B Ankle Fracture Treatment: a Combined Randomised and Observational Study (CROSSBAT)
}

\author{
Rajat Mittal, Ian A Harris, Sam Adie, Justine M Naylor, for the CROSSBAT \\ Study Group
}

To cite: Mittal R, Harris IA, Adie $\mathrm{S}$, et al. Surgery for Type B Ankle Fracture Treatment: a Combined Randomised and Observational Study (CROSSBAT). BMJ Open 2017;7:e013298.

doi:10.1136/bmjopen-2016013298

- Prepublication history and additional material is available. To view please visit the journal (http://dx.doi.org/ 10.1136/bmjopen-2016013298).

Received 13 July 2016 Revised 10 January 2017 Accepted 20 February 2017

CrossMark

Orthopaedic Department Whitlam Orthopaedic Research Centre, Ingham Institute for Applied Medical Research, South Western Sydney Clinical School, UNSW Australia, Liverpool BC, New South Wales, Australia

Correspondence to Dr Rajat Mittal; rajatmittal.syd@gmail.com

\section{ABSTRACT}

Background: Isolated type B ankle fractures with no injury to the medial side are the most common type of ankle fracture.

Objective: This study aimed to determine if surgery is superior to non-surgical management for the treatment of these fractures.

Methods: A pragmatic, multicentre, single-blinded, combined randomised controlled trial and observational study. Setting Participants between 18 and 65 years with a type $B$ ankle fracture and minimal talar shift were recruited from 22 hospitals in Australia and New Zealand. Participants willing to be randomised were randomly allocated to undergo surgical fixation followed by mobilisation in a walking boot for 6 weeks. Those treated non-surgically were managed in a walking boot for 6 weeks. Participants not willing to be randomised formed the observational cohort. Randomisation stratified by site and using permuted variable blocks was administered centrally. Outcome assessors were blinded for the primary outcomes. Primary outcomes Patient-reported ankle function using the American Academy of Orthopaedic Surgeons Foot and Ankle Outcomes Questionnaire (FAOQ) and the physical component score (PCS) of the SF-12v2 General Health Survey at 12 months postinjury. Primary analysis was intention to treat; the randomised and observational cohorts were analysed separately.

Results: From August 2010 to October 2013, 160 people were randomised (80 surgical and 80 nonsurgical); 139 (71 surgical and 68 non-surgical) were analysed as intention to treat. 276 formed the observational cohort (19 surgical and 257 nonsurgical); 220 (18 surgical and 202 non-surgical) were analysed. The randomised cohort demonstrated that surgery was not superior to non-surgery for the FAOQ (49.8 vs 53.0 ; mean difference $3.2(95 \% \mathrm{Cl} 0.4$ to $5.9), p=0.028$ ), or the PCS (53.7 vs 53.2 ; mean difference $0.6(-2.9$ to 1.8$), p=0.63) .23(32 \%)$ and 10 $(14 \%)$ participants had an adverse event in the surgical and non-surgical groups, respectively. Similar results were found in the observational cohort.

Conclusions: Surgery is not superior to non-surgical management for 44-B1 ankle fractures in the short term, and is associated with increased adverse events. Trial registration number: NCT01134094.

\section{Strengths and limitations of this study}

- The strengths of CROSSBAT (Combined Randomised and Observational Study of Surgery for type B Ankle fracture Treatment) include allocation concealment.

- In the randomised cohort, loss to follow-up and cross-over rates were low, and the as-treated analysis supported the findings of the intention-to-treat analysis.

- Outcome tools were validated and relevant, and assessors were blinded.

- The addition of the observational arm added to generalisability of the findings and addressed selection bias.

- Limitations include the lack of blinding of the surgeons and participants which is unavoidable with this trial design and the use of subjective scoring only.

Ankle fractures are common, with 1 in 800 people fracturing their ankle every year. ${ }^{1-3}$ The most common pattern involves a fracture of the distal fibula (lateral malleolus) at the level of the tibiofibular syndesmosis, otherwise known as an Association for the Study of Internal Fixation (AO) or Orthopaedic Trauma Association (OTA) type $\mathrm{B}$ ankle fracture. ${ }^{4-7}$ If combined with displacement of the ankle mortise or a fracture of the medial malleolus, surgical fixation is the preferred treatment. However, the most common type of ankle fracture involves a type B lateral malleolus fracture without fracture of the medial malleolus or displacement of the talus (AO/OTA-type 44-B1).

Management options for these AO 44-B1 ankle fractures include surgical stabilisation by internal fixation using a plate and screws or non-surgical management using a cast or a walking boot. ${ }^{1}$ Advocates for surgical management emphasise the importance of achieving an anatomic reduction with internal fixation, thereby limiting the potential for displacement and instability. ${ }^{9}$ 
Advocates for non-surgical management argue that functional outcomes are not superior to surgical stabilisation and surgery is associated with significant costs and possible adverse events. ${ }^{8}{ }^{10-12}$ These include the general risks of anaesthesia and surgery, such as death, venous thromboembolism, infection, failure of fixation and the need for revision surgery. ${ }^{12}$ Slobogean et al ${ }^{13}$ showed that the average costs of non-surgical and surgical management of an unstable, isolated, lateral malleolar fracture were US $\$ 1892$ and US $\$ 6404$, respectively.

A national survey of 358 orthopaedic surgeons in Australia revealed that surgical management of this common fracture is preferred by $\sim 40 \%$ of surgeons, despite a lack of evidence to support this approach. ${ }^{14}$ Recognising the costs and risks associated with surgery, the lack of evidence supporting the benefit of surgery and the considerable practice variation, we designed a randomised trial to determine the comparative effectiveness of surgical and non-surgical management.

In this study involving participants with a 44-B1 ankle fracture, we sought to determine whether surgical management provided superior ankle function and quality of life at 12 months postinjury when compared with nonsurgical management. A concurrent observational cohort study was included to provide further evidence regarding the outcomes obtained in routine practice and to improve the generalisability of the results.

\section{METHODS}

Study design

CROSSBAT (Combined Randomised and Observational Study of Surgery for type B Ankle fracture Treatment) was a pragmatic, multicentre, parallel-group, superiority, randomised controlled trial with an observational cohort that recruited participants from August 2010 to October 2013. It involved 22 hospitals in Australia and New Zealand that were a mix of rural, regional and metropolitan centres (a list of recruiting hospitals is provided in the online supplementary appendix). The main study was the randomised group, and participants declining randomisation were invited to participate in the observational cohort. The protocol was approved by the ethics committees relevant for each site. The full protocol can be accessed as an online supplementary material on the $B M J$ website.

\section{Participants}

Consecutive adult patients presenting to a recruiting hospital during the study period with an isolated, closed AO-type 44-B1 distal fibula fracture without significant talar shift presenting within 10 days of injury were screened for eligibility. Significant talar shift was defined as medial clear space being at least $2 \mathrm{~mm}$ wider than the superior clear space on a mortise X-ray view of the ankle. Further inclusion criteria were patients aged between 18 and 65 years inclusive with no other concomitant fractures/dislocations; mobilising unaided/independently preinjury; and willing to be followed up for 12 months. Exclusion criteria were participants who were medically unfit for anaesthesia/surgery; skeletally immature; previous trauma or surgery to the fractured ankle; inability to consent; pregnancy; the presence of comorbidities that impede mobilisation; and non-English speaking. Written informed consent was obtained from all patients willing to participate.

\section{Randomisation and blinding}

Eligible participants willing to be randomised were randomly allocated in a 1:1 ratio to either the surgical or non-surgical intervention. The National Health and Medical Research Council Clinical Trials Centre (not otherwise involved in the study) generated the randomisation schedule using a permuted block approach with variable block size and stratified by site. Randomisation was administered using an automated telephone-based system that provided allocation concealment. Owing to the nature of the interventions, neither the investigators nor the participants were blinded. Outcome assessors were independent of the treating teams, and collected data using a standardised telephone interview. As part of the opening conversation, patients were advised not to disclose their treatment so that the assessor could remain blind to treatment. After randomisation, the surgical group received surgery within 10 days of injury. Eligible participants who declined randomisation were invited to enter the observational cohort. Treatment for the observational cohort was determined by participant and surgeon preference.

\section{Procedures}

During protocol development, members of the Australian Orthopaedic Trauma Society were consulted regarding the best practice for the surgical and nonsurgical management of 44-B1 ankle fractures as well as the primary and secondary outcomes. Patient eligibility centred on the presence of the fracture of interest. An external rotation stress test to assess the stability of the ankle was not performed as it was not routine practice in Australia owing to uncertainty about its validity and clinical utility. ${ }^{15} 16$ The focus for the effectiveness of the interventions was patient-reported outcomes. Radiological measures beyond 6 weeks were not required as they were unlikely to demonstrate any osteoarthritic changes and because late malalignment was considered rare (with both methods of treatment) and unlikely to influence management without clinical symptoms. One recruiting site declined to randomise participants due to lack of equipoise within the orthopaedic department and contributed to the observational cohort only.

The technique for surgical management was surgical fixation using a plate and screws. Surgeries were performed by orthopaedic surgeons or by orthopaedic trainees under the supervision of consultant orthopaedic surgeons following the AO principles of fracture fixation. Plate placement and reduction techniques were 
left to the discretion of the surgeon. Adverse intraoperative or postoperative events were recorded. Postoperatively, all participants were non-weight bearing and placed in a below-knee plaster cast or walking boot. Discharge from hospital was determined by the participant's ability to walk $25 \mathrm{~m}$ unaided with standby assistance as determined by a physiotherapist (usual discharge criteria). The treating surgeon reviewed the participant after 10-14 days for wound assessment and change of cast to a walking cast or a walking boot (cam walker). The participant was then allowed full weight bearing. The treating surgeon reviewed the participant 6 weeks postinjury with ankle radiographs and removed the cast or walking boot.

Participants who were treated non-surgically were managed with a walking boot and allowed full weight bearing. Discharge from hospital was determined as for the surgical group. All participants were examined within 10-14 days postinjury by the treating surgeon who assessed the patient with new ankle radiographs. The treating surgeon reviewed the participant 6 weeks postinjury with repeat ankle radiographs and removed the cast or walking boot.

Referral to physiotherapy for all participants was at the discretion of the treating surgeon.

\section{Outcomes}

The primary outcome measures were patient-reported ankle function using the American Academy of Orthopaedic Surgeons Foot and Ankle Outcomes Questionnaire (FAOQ) and the health-related quality of life using the physical component score (PCS) of the SF-12v2 General Health Survey at 12 months postinjury. The FAOQ is a validated, patient-reported outcome measure that assesses ankle function with a higher value indicating better function. ${ }^{17}{ }^{18}$ Normative FAOQ scores were used, with a score of 50 representing the mean in the general population and an SD of $10 .{ }^{19}$ Similarly, the $\mathrm{SF}-12 \mathrm{v} 2$ is a validated patient-reported outcome measure that has been used for the assessment of people with ankle fractures, with a higher value indicating better health. ${ }^{20-22}$ Both the SF-12v2 and the FAOQ have been used previously for patients with ankle fractures. ${ }^{22} 23$ Secondary end points included any adverse events in the 12 months postinjury; return to work at 6 weeks and 3, 6 and 12 months postinjury; the PCS and FAOQ at 3 and 6 months postinjury; and the mental component score of the SF-12v2 at 3, 6 and 12 months postinjury. Adverse events were classified as major (unplanned/repeat surgery; infection requiring admission to hospital; pulmonary embolus or death) or minor (neurological injury not requiring further intervention; infections not requiring hospital admission; deep vein thrombosis or other adverse events not requiring hospital admission or surgery). ${ }^{24}$ The adverse events were collected at 6 weeks and 3, 6 and 12 months postinjury. Follow-up assessments were conducted by telephone. Physiotherapy use (number of visits) was measured.

\section{Statistical analysis}

The PCS has an SD of 10 points and a 5-point difference (equivalent to a $0.5 \mathrm{SD}$ ) is considered to be the minimum clinically important difference. ${ }^{20} \quad 21 \quad 25 \mathrm{~A}$ sample size of 160 in the randomised cohort was used to provide $80 \%$ power to detect a five-point difference in the PCS between the two groups at a significance level of 0.05 , allowing for a $20 \%$ loss to follow-up. The normative FAOQ score has an SD of 10, with a 5-point difference $(0.5 \mathrm{SD})$ regarded as the minimum clinically important difference. ${ }^{19}$ The same sample size (160) would provide the same power to detect a $0.5 \mathrm{SD}$ difference in the FAOQ. There was no sample size target for the observational cohort as this cohort was to provide online supplementary information for the randomised cohort. The randomised and observational cohorts were analysed separately. The primary analysis, conducted using intention-to-treat principles, was performed on the randomised cohort; an as-treated analysis was also performed on the randomised cohort for sensitivity testing. Normality was assessed and Student's t-test was used to compare continuous variables between groups. Missing data were not imputed. The $\chi^{2}$ or Fisher's exact test was used for categorical data analysis as appropriate. Statistical analysis was conducted using SAS V.9.4 (Cary, North Carolina, USA). Both primary outcomes were required to be significantly better in the surgical arm in order for surgery to be regarded as superior. The trial was registered with clinicaltrials.gov (NCT01134094).

\section{Patient involvement}

Patients were involved in the development of the outcome measures. ${ }^{17}$ 19-21 They were not involved in the development or conduct of the study. Publication details will be disseminated to study participants who expressed an interest in knowing the results of this study. All participants were thanked in Acknowledgements statement for participating in this study. The burden of intervention on patients was assessed and considered to be low by the ethics committee that assessed the research project (given that both the intervention and control arms are routine practice); no patients were involved in that assessment. This was done as part of a survey of patient factors influencing participation in surgical randomised trials embedded within CROSSBAT. ${ }^{26}$

\section{Role of the funding source}

This trial was supported in part by a grant from the Australian Orthopaedic Association Research Foundation. RM was supported with: a postgraduate scholarship from the National Health and Medical Research Council, Avant Doctors-in-training research scholarship and the Foundation for Surgery John Loewenthal Research Fellowship from the Royal Australasian College of Surgeons. The funding organisations of the study had no role in the study design, data collection, data analysis, data interpretation or writing of the report. The corresponding author had full access to 
all the data in the study and had final responsibility for the decision to submit for publication.

\section{RESULTS}

From 15 August 2010 to 3 October 2013, 436 participants who presented with an isolated, closed AO-type 44-B1 distal fibula fracture with minimal talar shift were screened and all were recruited; 160 participants were randomised to the randomised cohort and all 276 participants who declined randomisation were included in the observational cohort. The cohort ascertainment and retention flow chart is presented in figure 1.

In the randomised cohort, 80 participants were randomised to non-surgical management and 80 were randomised to surgical management. At 12 months, 68 (85\%) and $71(89 \%)$ participants were followed up in the nonsurgical and surgical groups, respectively. The intention-to-treat analysis kept participants in the groups to which they were randomised, but the numbers are incomplete due to missing data.

In the observational cohort, 257 participants were treated non-surgically and 19 were treated surgically as most patients declined surgery when informed of equipoise regarding the two treatment arms. At 12 months, $202(79 \%)$ participants were followed up in the nonsurgical group, and $18(95 \%)$ participants were followed up in the surgical group.

Baseline participant characteristics were similar between the two groups in the randomised cohort. In the observational cohort, the surgical group was significantly younger than the non-surgical group (mean difference $8.3,95 \%$ CI 2.6 to $14.0 ; \mathrm{p}=0.007$ ). There were no other significant differences in baseline demographics between the two groups in the observational cohort. Baseline characteristics are shown in table 1 . Comparison of baseline data between the randomised

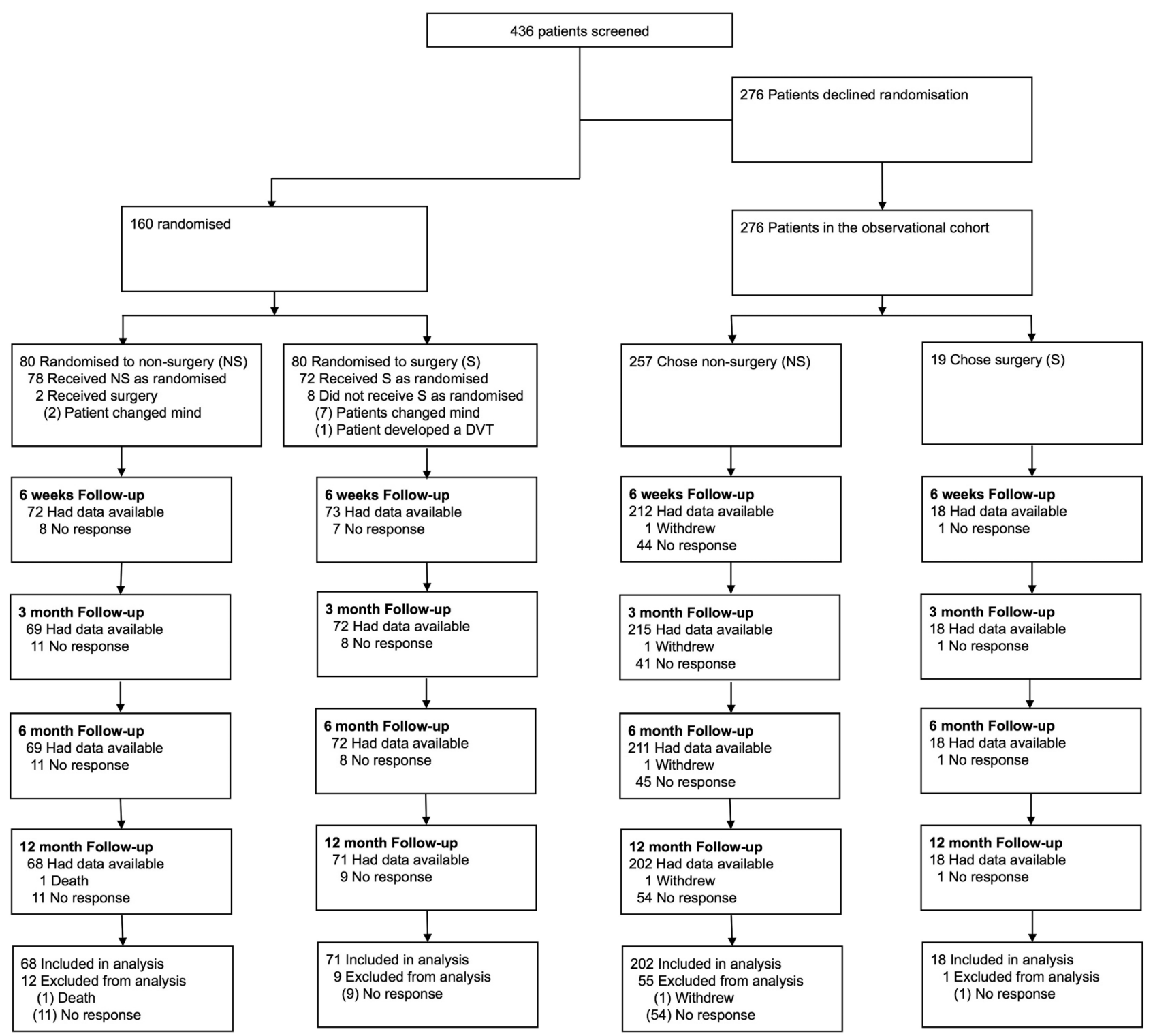

Figure 1 Cohort ascertainment and retention. DVT, deep vein thrombosis. 
Table 1 Baseline demographics for CROSSBAT

\begin{tabular}{|c|c|c|c|c|}
\hline \multirow[b]{2}{*}{ Variable } & \multicolumn{2}{|c|}{ Randomised cohort } & \multicolumn{2}{|c|}{ Observational cohort } \\
\hline & Surgical $(n=80)$ & Non-surgical $(n=80)$ & Surgical $(n=19)$ & Non-surgical $(n=257)$ \\
\hline Age, mean (SD), years & $38.1(13.0)$ & $39.8(13.7)$ & $31.1(11.5)^{\star}$ & $39.4(13.7)^{\star}$ \\
\hline Female, $\mathrm{n}(\%)$ & $42(53)$ & $41(51)$ & $5(26)$ & $115(45)$ \\
\hline BMI, mean (SD), $\mathrm{kg} / \mathrm{m}^{2}$ & $27.7(5.2)$ & $28.4(6.6)$ & $26.2(2.9)$ & $27.6(5.5)$ \\
\hline Left side, $\mathrm{n}(\%)$ & $41(51)$ & $46(58)$ & $11(58)$ & $120(47)$ \\
\hline \multicolumn{5}{|l|}{ Mechanism, n (\%) } \\
\hline Fall $<1 \mathrm{~m}$ & $70(90)$ & $67(84)$ & $17(90)$ & $232(92)$ \\
\hline Fall $>1 \mathrm{~m}$ & $6(8)$ & 8 (10) & $0(0)$ & $9(4)$ \\
\hline Motor vehicle accident & $2(3)$ & $5(6)$ & $2(11)$ & $11(5)$ \\
\hline \multicolumn{5}{|l|}{ Education, n (\%) } \\
\hline High school or lower & $31(39)$ & $44(55)$ & $11(58)$ & $100(39)$ \\
\hline TAFE/diploma & $30(38)$ & $23(29)$ & $4(21)$ & $78(30)$ \\
\hline University or above & $17(21)$ & $12(15)$ & $4(21)$ & $73(29)$ \\
\hline Diabetes mellitus, n (\%) & $3(4)$ & $4(5)$ & $0(0)$ & $10(4)$ \\
\hline Peripheral vascular disease, $\mathrm{n}(\%)$ & $1(1)$ & $0(0)$ & $0(0)$ & $1(1)$ \\
\hline Alcohol, n (\%)† & $60(78)$ & $63(79)$ & $15(79)$ & 177 (69) \\
\hline Smoker, n (\%)‡ & $29(36)$ & $28(35)$ & $9(47)$ & $74(29)$ \\
\hline Working, n (\%) & $64(80)$ & $65(81)$ & $15(79)$ & $197(77)$ \\
\hline \multicolumn{5}{|l|}{ Insurance status, n (\%) } \\
\hline Public & $50(63)$ & $57(71)$ & $7(37)$ & $160(63)$ \\
\hline Private & $18(23)$ & $19(24)$ & $9(47)$ & $75(30)$ \\
\hline Compensation & $10(13)$ & $3(4)$ & $3(16)$ & $18(7)$ \\
\hline
\end{tabular}

*Surgical group was significantly younger than non-surgical group in the observational cohort $(p=0.007)$.

†A patient was described as consuming alcohol if they were drinking one or more standard drinks per month.

$\ddagger A$ patient was described as a smoker if they were smoking one or more cigarettes per month.

BMI, body mass index; CROSSBAT, Combined Randomised and Observational Study of Surgery for type B Ankle fracture Treatment; TAFE,

Technical and Further Education.

and observational cohorts showed that both cohorts had similar demographic profiles.

For the randomised cohort, at 12 months, intention-to-treat analysis demonstrated that the surgical group was not superior to the non-surgical group. With respect to the FAOQ there was a statistically significant difference favouring the non-surgical group (mean difference $3.2 ; 95 \%$ CI 0.4 to $5.9 ; \mathrm{p}=0.028$ ), but this difference was not clinically meaningful. The minimum and maximum values of FAOQ scores were 5.8-55.6 and 32.6-55.6 for the surgical and non-surgical groups, respectively. The surgical group was not superior to the non-surgical group with respect to the PCS (mean difference 0.6, favouring the non-surgical group; $95 \% \mathrm{CI}-2.9$ to $1.8 ; \mathrm{p}=0.63$ ). The surgical group had a significantly higher proportion of participants with overall adverse events (risk ratio $(\mathrm{RR})=2.3 ; 95 \%$ CI 1.2 to $5.4 ; \mathrm{p}=0.01$ ) and minor adverse events $(\mathrm{RR}=2.9 ; 95 \%$ CI 1.3 to 6.4 ; $\mathrm{p}=0.009)$. No significant differences in the proportion of participants with major adverse events were found $(\mathrm{RR}=2.0 ; 95 \%$ CI 0.5 to $7.8 ; \mathrm{p}=0.30)$. A breakdown of the adverse events is provided in the online supplementary appendix. There was one death in the non-surgical group. This participant was an intravenous drug user who overdosed and died between 6 and 12 months postinjury. The length of hospital stay was shorter in the non-surgical group (mean difference 1.5 days; $95 \%$ CI 0.9 to $2.0 ; \mathrm{p}<0.001)$. A significantly higher proportion of participants from the surgical group used outpatient physiotherapy $(\mathrm{RR}=1.5 ; 95 \%$ CI 1.1 to $2.2 ; \mathrm{p}=0.01)$. There was no significant difference between the surgical and non-surgical groups with respect to the proportion of participants (of those who were working preinjury) returning to work at 6 weeks ( $\mathrm{RR}=0.87 ; 95 \%$ CI 0.62 to 1.2; $\mathrm{p}=0.41$ ). A summary of the outcomes is presented in table 2 and figure 2.

There were 10 protocol violations; 8 patients randomised to the surgical group were treated non-surgically (7 later declined surgery; 1 was diagnosed with a deep vein thrombosis presurgery) and 2 patients randomised to the non-surgical group were treated surgically due to protocol violations by treating surgeons.

An as-treated analysis of the randomised cohort was also conducted. It also showed that the surgical group was not superior to the non-surgical group for any outcomes. These results are presented in the online supplementary appendix. Results for the observational cohort are presented in the online supplementary appendix as well.

\section{DISCUSSION}

\section{Principal findings}

In adult patients aged from 18 to 65 years with an isolated type B ankle fracture with minimal talar shift, surgical management was not superior to non-surgical management in terms of ankle function and health-related quality of life at 12 months postinjury. Furthermore, surgical management was not superior to 
Table 2 Results for the intention-to-treat analysis

\begin{tabular}{|c|c|c|c|c|}
\hline \multirow{2}{*}{$\begin{array}{l}\text { Variable } \\
3 \text { months }\end{array}$} & \multicolumn{4}{|c|}{ Randomised cohort (intention-to-treat analysis) } \\
\hline & $\begin{array}{l}\text { Surgical } \\
\mathrm{n}=72\end{array}$ & $\begin{array}{l}\text { Non-surgical } \\
\mathrm{n}=69\end{array}$ & Difference $(95 \% \mathrm{Cl})$ & p Value \\
\hline FAOQ, mean (SD) & $43.8(12.0)$ & $44.7(12.2)$ & $0.9(-3.1 \text { to } 5.0)^{*}$ & 0.65 \\
\hline PCS, mean (SD) & $47.1(10.5)$ & $46.8(11.6)$ & $0.24(-3.9 \text { to } 3.5)^{*}$ & 0.90 \\
\hline MCS, mean (SD) & $55.0(10.3)$ & $56.4(7.4)$ & $1.4(-1.6 \text { to } 4.4)^{*}$ & 0.37 \\
\hline Working, n (\%)† & $55 / 64(86 \%)$ & $57 / 61(93 \%)$ & $0.47(0.15$ to 1.4$) \ddagger$ & 0.17 \\
\hline 6 months & $\mathrm{n}=72$ & $n=69$ & & \\
\hline FAOQ, mean (SD) & $49.1(8.4)$ & $51.9(5.6)$ & $2.7(0.4 \text { to } 5.1)^{\star}$ & 0.025 \\
\hline PCS, mean (SD) & $50.4(8.9)$ & $52.3(7.4)$ & $1.9(-0.90 \text { to } 4.6)^{*}$ & 0.18 \\
\hline MCS, mean (SD) & $56.6(7.2)$ & $57.2(7.9)$ & $0.6(-2.0 \text { to } 3.1)^{\star}$ & 0.66 \\
\hline Working, $\mathrm{n}(\%) \dagger$ & $62 / 63(98)$ & $61 / 61(100)$ & NA & 1.00 \\
\hline 12 months & $\mathrm{n}=71$ & $\mathrm{n}=68$ & & \\
\hline FAOQ, mean (SD) & $49.8(10.6)$ & $53.0(5.2)$ & $3.2(0.4 \text { to } 5.9)^{*}$ & 0.028 \\
\hline PCS, mean (SD) & $53.7(7.1)$ & $53.2(6.7)$ & $0.6(-1.8 \text { to } 2.9)^{*}$ & 0.63 \\
\hline MCS, mean (SD) & $55.2(11.1)$ & $56.5(9.7)$ & $1.3(-2.2 \text { to } 4.8)^{*}$ & 0.47 \\
\hline Working, n (\%)† & $62 / 63(98)$ & $60 / 60(100)$ & NA & 1.00 \\
\hline Any adverse event, $n(\%)$ & 23/73 (32) & $10 / 74(14)$ & 2.3 (1.2 to 4.5$) \ddagger$ & 0.009 \\
\hline Major adverse event, n (\%) & 6/73 (8) & $3 / 74(4)$ & $2.0(0.5$ to 7.8$) \ddagger$ & 0.33 \\
\hline Minor adverse event, $\mathrm{n}(\%)$ & $20 / 73(27)$ & $7 / 74(10)$ & $2.8(1.3$ to 6.4$) \ddagger$ & 0.006 \\
\hline Physiotherapy use, n (\%) & $44 / 73(60)$ & 28/72 (39) & 1.5 (1.1 to 2.2$) \ddagger$ & 0.010 \\
\hline
\end{tabular}

non-surgical management for any secondary outcomes and it was associated with a longer length of hospital stay and a higher rate of adverse events.

CROSSBAT was a randomised controlled trial with a parallel observational cohort. The randomised cohort provides a robust comparison of effectiveness between the two treatment groups while the observational cohort provides a concurrent cohort subjected to routine clinical practice. The two cohorts had largely similar baseline characteristics indicating that the results of the randomised trial are generalisable to similar patients who decline randomisation. Further details of baseline comparisons are provided in the appendix. For these reasons, we believe that dissemination of the results of CROSSBAT will help address the practice variation that exists in this area. ${ }^{1427}$

\section{Comparison with other studies}

A recent systematic review conducted by Donken et al ${ }^{1}$ showed that there was insufficient evidence to justify surgical management of type $B$ ankle fractures. This is because the prevailing RCTs identified by the review included patients with either different patterns of ankle fractures and/or with significant talar shift that potentially confounds the need for surgery. ${ }^{728-32}$ A recent study consented 81 patients to either surgical or nonsurgical management for potentially unstable type B ankle fractures (type B ankle fractures that had a positive external rotation stress test indicating a significant lateral talar shift). ${ }^{33}$ Despite the presence of slight talar misalignment in $20 \%$ of the non-surgical group at 1 year, patients managed surgically did not have superior functional outcomes to those managed non-surgically. ${ }^{33}$ It is possible that a minority of patients in the nonsurgical group studied within CROSSBAT also had some misalignment at 1 year, but it was likely to have been subclinical given the good clinical scores. To assess the longer term implications of surgical and non-surgical management of these ankle fractures, we plan to conduct longer term follow-up of the participants using both radiographic and functional measures.

\section{Strengths and limitations}

The strengths of CROSSBAT include allocation concealment, which was assured through employment of a third party overseeing randomisation and allocation. In the randomised cohort, loss to follow-up and cross-over rates were low, and the as-treated analysis supported the findings of the intention-to-treat analysis. Outcome tools were validated and relevant, and assessors were blinded. The addition of the observational arm added to generalisability of the findings and addressed selection bias.

Limitations include the lack of blinding of the surgeons and participants which is unavoidable with this trial design. It is also possible that some eligible participants were missed, as recruitment fluctuated over time and between sites, given that dedicated research officers were not present at the sites due to funding constraints. However, all participants who were approached were willing to be recruited to either the randomised or 

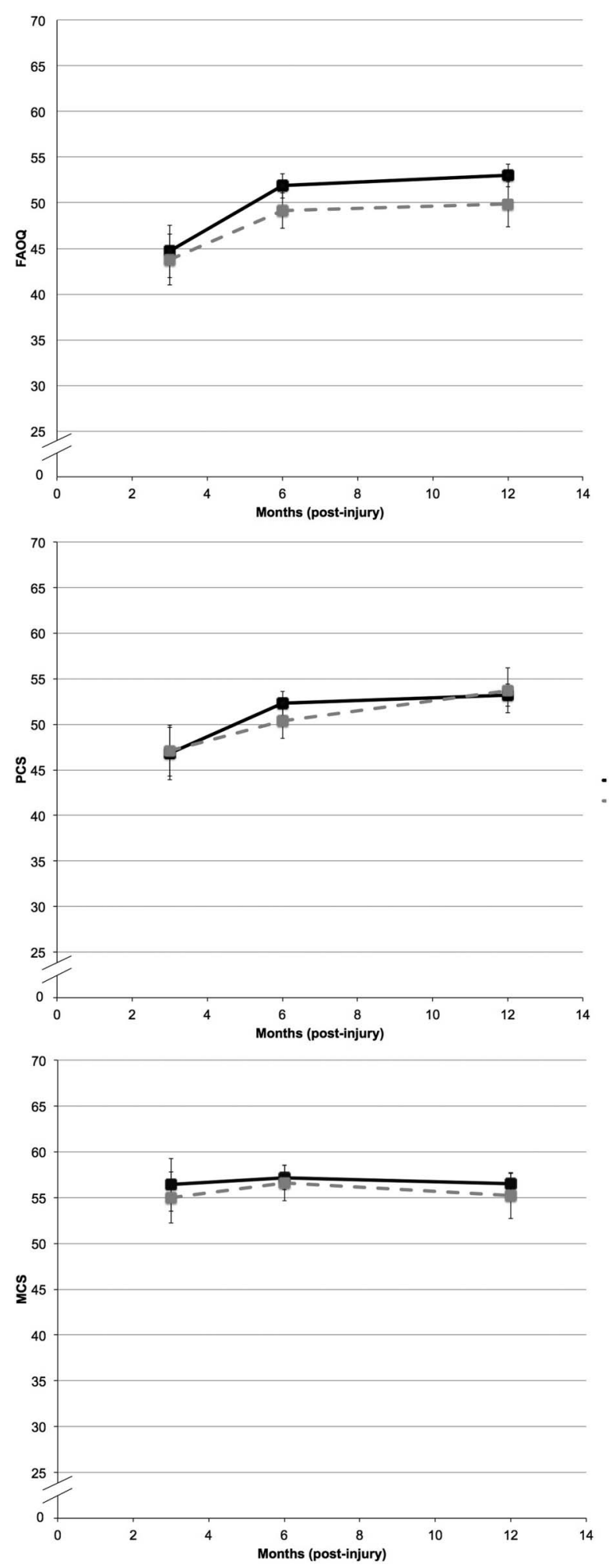

Figure 2 Differences between surgical and non-surgical groups with respect to ankle function and general health for the randomised cohort. FAOQ, PCS and MCS are the scores of the SF-12v2 general health survey for the randomised and cohort. Higher value represents better function. Error bars represent $95 \% \mathrm{Cl}$. Solid black line represents the non-surgical group while the dashed grey line represents the surgical group. FAOQ, American Academy of Orthopaedic Surgeons Foot and Ankle Outcomes Questionnaire; MCS, mental component scores; PCS, physical component scores. observational cohort. The physiotherapy practices postinjury were not controlled, as participants were free to access physiotherapy services as desired. It was noted that a higher proportion of participants managed surgically sought physiotherapy. This, however, did not result in improved patient-reported outcomes for the surgical group. Further, a recent review by Lin Chung-Wei $e t a l^{34}$ showed no evidence of improved outcomes with physiotherapy-based rehabilitation following ankle fractures. Some may consider the use of subjective scoring to be a limitation; however, both the SF-12v2 and the FAOQ have been validated and used previously for patients with ankle fractures. ${ }^{22}{ }^{23}$ It can also be argued that clinical decisions about treating patients should be based on symptoms rather than radiographs. Although this study presents 1-year results, future research would include further follow-up of this cohort to assess the longer term effect of surgical and non-surgical management of these 44-B1 ankle fractures.

\section{CONCLUSION}

The results of this study demonstrate that surgical management is not superior to non-surgical management in type B ankle (fibula) fractures with minimal talar shift in the short term and is associated with increased adverse events. Further follow-up is needed to assess the difference between the two groups in the longer term.

Acknowledgements The authors would like to thank all the participants who participated in this study.

Collaborators CROSSBAT Study Group: SA, Liverpool Hospital, New South Wales, Australia, sam.adie@gmail.com; Paul Allcock, Lyell McEwin Hospital, South Australia, Australia, Paul.Allcock@health.sa.gov.au; Mustafa Alttahir, Campbelltown Hospital, New South Wales, Australia, Mustafa.alttahir@gmail. com; Zsolt Balogh, John Hunter Hospital, New South Wales, Australia, Zsolt. Balogh@hnehealth.nsw.gov.au; Aziz Bhimani, Wollongong Hospital, New South Wales, Australia, azizb@mac.com; Russell Bourne, Nambour Hospital, Queensland, Australia, bourner@bigpond.com; Richard Boyle, Royal Prince Alfred Hospital, New South Wales, Australia, rich_boyle@hotmail.com; David Broe, Prince of Wales Hospital, New South Wales, Australia, davebroe@lycos. com; Andrew Bucknill, Royal Melbourne Hospital, Victoria, Australia, andrew. bucknill@mh.org.au; Mellick Chehade, Royal Adelaide Hospital, South Australia, Australia, mellick.chehade@adelaide.edu.au; Raymond Chin, Liverpool Hospital, New South Wales, Australia, ray.chin@iinet.net.au; Andrew Clout, Campbelltown Hospital, New South Wales, Australia, a.j.clout@gmail. com; Frank Connon, St George Hospital, New South Wales, Australia, francisconnon@gmail.com; Cameron Cooke, Princess Alexandra Hospital, Queensland, Australia, cameroncooke@lowerlimb.com.au; Chandra Dave, Liverpool Hospital, New South Wales, Australia, chandradave61@gmail.com; Jaykar Dave, Liverpool Hospital, New South Wales, Australia, orthopod@optusnet.com.au; Mark Dekkers, Princess Alexandra Hospital, Queensland, Australia, mark_dekkers@health.qld.gov.au; Herwig Drobetz, Mackay Base Hospital, Queensland, Australia, drobo@aon.at; Richard Farrugia, Royal Melbourne Hospital, Victoria, Australia, richard.farrugia@mh.org.au; Brett Fritsch, Royal Prince Alfred Hospital, New South Wales, Australia, brett@brettfritsch.com.au; Sanjeev Gupta, Royal Prince Alfred Hospital, New South Wales, Australia, sanjeev.gupta@bigpond.com; IAH, Liverpool Hospital, New South Wales, Australia, iaharris1@gmail.com; Chris Hoffman, Wellington Hospital, Wellington, New Zealand, cwhoffman@xtra.co.nz; Michael Holt, Royal Brisbane Hospital, Queensland, Australia, mjholt@wesportsmed.com.au; Mark Horseley, Royal Prince Alfred Hospital, New South Wales, Australia, drmhorsley@gmail.com; Stephen Hutchinson, Prince of Wales Hospital, New South Wales, Australia, shutchin1978@gmail.com; Prajith Jeyaprakash, 
University of New South Wales, New South Wales, Australia, prajithjeyaprakash@gmail.com; Steven Kent, Wollongong Hospital, New South Wales, Australia, skent1985@gmail.com; Doug King, Princess Alexandra Hospital, QueensInd, Australia, Iloyd_king@health.qld.gov.au; Victoria Ko, Liverpool Hospital, New South Wales, Australia, vwmko@yahoo.com; Vinay Kulkarni, Campbelltown Hospital, New South Wales, Australia, vinay@doctor. id.au; Martin Laird, Liverpool Hospital, New South Wales, Australia, mlaird@gmp.usyd.edu.au; David Lieu, Liverpool Hospital, New South Wales, Australia, david.t.lieu@bigpond.com; Andreas Loefler, Prince of Wales Hospital, New South Wales, Australia, andreasloefler@optusnet.com.au; David Lunz, Prince of Wales Hospital, New South Wales, Australia,

footandanklesurgery@yahoo.com; Genni Lynch, Princess Alexandra Hospital, Queensland, Australia, genni.lynch@health.qld.gov.au; Lawrie Malisano, Princess Alexandra Hospital, Queensland, Australia, I.malisano@uq.edu.au; Ian Meakin, Bankstown Hospital, New South Wales, Australia, imeakin@bigpond. net.au; Robert Molnar, Westmead Hospital, New South Wales, Australia, rmolnar@optusnet.com.au; Chris Morrey, Cairns Base Hospital, Queensland, Australia, cmorrey@coc.net.au; Jonathan Mulford, Prince of Wales Hospital, New South Wales, Australia, jonathanmulford1971@gmail.com; Ashish Munsif, University of New South Wales, New South Wales, Australia, z3288258@zmail.unsw.edu.au; Paul Muscio, Cairns Base Hospital, Queensland, Australia, paul_muscio@health.qld.gov.au; Alok Narayan, Wollongong Hospital, New South Wales, Australia, rahintern@yahoo.com.au; Alexander Nicholls, Canberra Hospital, Australian Capital Territory, Australia, alexandernicholls@gmail.com; JMN, University of New South Wales, New South Wales, Australia, Justine.Naylor@sswahs.nsw.gov.au; Fred Nouh, Campbelltown Hospital, New South Wales, Australia, frednouh@yahoo.com.au; Tim 0'Carrigan, Campbelltown Hospital, New South Wales, Australia, Dr. Ocarrigan@sbjc.com.au; Ed O'Leary, St George Hospital, New South Wales, Australia, edmundoleary@hotmail.com; Peter Lorentzos, Westmead Hospital, New South Wales, Australia, peter.Iorentzos@gmail.com; Sushil Pant, St George Hospital, New South Wales, Australia, sushpant@gmail.com; Diana Perriman, Canberra Hospital, Australian Capital Territory, Australia, Diana. perriman@act.gov.au; Jeffrey Petchell, Royal Prince Alfred Hospital, New South Wales, Australia, jfpetchell@aapt.net.au; Marinis Pirpiris, Royal Melbourne Hospital, Victoria, Australia, surgeons@orthopaedicsvictoria.com. au; Tony Pohl, Royal Adelaide Hospital, South Australia, Australia, anthony. pohl@adelaide.edu.au; Vaibhav Punjabi, Liverpool Hospital, New South Wales, Australia, vaibhav_punjabi@yahoo.com; Sunil Randhawa, Liverpool Hospital, New South Wales, Australia, sunil.randhawa@ausdoctors.net; Matthias Rau, Rockhampton Hospital, Queensland, Australia, gainsgorn@yahoo.com.au; Jennie Scarvell, Canberra Hospital, Australian Capital Territory, Australia, Jennie.Scarvell@canberra.edu.au; Michael Schuetz, Princess Alexandra Hospital, Queensland, Australia, m.schuetz@qut.edu.au; Ben Scwartz, Wollongong Hospital, New South Wales, Australia, drbenschwarz@gmail.com; Paul Smith, Canberra Hospital, Australian Capital Territory, Australia, psmith. admin@orthoact.com.au; Brahman Sivakumar, Campbelltown Hospital, New South Wales, Australia, brahman.sivakumar@gmail.com; Bogdan Solomon, Royal Adelaide Hospital, South Australia, Australia, bogdan.solomon@health. sa.gov.au; Jonathan Spencer, Sir Charles Gairdner Hospital, Western Australia, Australia, jonmfspencer@gmail.com; Paul Stalley, Royal Prince Alfred Hospital, New South Wales, Australia, pdstalley@optusnet.com.au; Mitchell Steele, Wollongong Hospital, New South Wales, Australia, mitchelljsteele@gmail.com; Mayuran Suthersan, Campbelltown Hospital, New South Wales, Australia, msuthersan@gmail.com; Zoltan Szomor, St George Hospital, New South Wales, Australia, zszomor@hotmail.com; Peter Tamblyn, Flinders Medical Centre, South Australia, Australia, Peter.Tamblyn@health.sa.gov.au; Seth Tarrant, John Hunter Hospital, New South Wales, Australia, sethtarrant@gmail. com; Kevin Tetsworth, Royal Brisbane Hospital, Queensland, Australia, kevin_tetsworth@health.qld.gov.au; Sameer Viswanathan, Campbelltown Hospital, New South Wales, Australia, sameerv@me.com; Richard Walker, Liverpool Hospital, New South Wales, Australia, drrmwalker@hotmail.com.

Contributors RM and IAH had full access to all of the data in the study and take responsibility for the integrity of the data and the accuracy of the data analysis and act as guarantors. RM, IAH, SA, JMN and CROSSBAT Study Group were involved in study, concept, design, acquisition, critical revision of the manuscript for important intellectual content. RM and IAH conducted statistical analysis and are responsible for the data analysis.
Funding This trial was supported in part by a grant from the Australian Orthopaedic Association Research Foundation. RM was supported by: a postgraduate scholarship from the National Health and Medical Research Council, Avant Doctors-in-training research scholarship and the Foundation for Surgery John Loewenthal Research Fellowship from the Royal Australasian College of Surgeons. None of the organisations had a direct role in the design and conduct of the study; collection, management, analysis, and interpretation of the data; preparation, review or approval of the manuscript; and decision to submit the manuscript for publication.

Competing interests None declared.

Ethics approval This study was approved by the following ethics committees:

- Central Regional Ethics Committee. Reference Number: CEN/12/06/030

- Ethics of Human Research Committee (TQEH and LMH). Reference Number: 2010130

- Flinders Clinical Research Ethics Committee. Reference Number: 358.10

- Hunter New England Human Research Ethics Committee. Reference Number: 09/12/16/5.02

- Melbourne Health Human Research Ethics Committee. Reference Number: 2010.027

- Metro South Human Research Ethics Committee. Reference Number: HREC/11/QPAH/145

- Royal Adelaide Hospital Research Ethics Committee. Reference Number: 100705

- Sir Charles Gairdner Group Human Research Ethics Committee. Reference Number: 2010-092

- University of New South Wales Human Research Ethics Committee. Reference Number: HC12187

Provenance and peer review Not commissioned; externally peer reviewed.

Data sharing statement Additional data from the study can be obtained from the corresponding author at rajatmittal.syd@gmail.com

Open Access This is an Open Access article distributed in accordance with the Creative Commons Attribution Non Commercial (CC BY-NC 4.0) license, which permits others to distribute, remix, adapt, build upon this work noncommercially, and license their derivative works on different terms, provided the original work is properly cited and the use is non-commercial. See: http:// creativecommons.org/licenses/by-nc/4.0/

\section{REFERENCES}

1. Donken CC, Al-Khateeb H, Verhofstad Michael HJ, et al. Surgical versus conservative interventions for treating ankle fractures in adults. Cochrane Database Syst Rev 2012;(8):CD008470.

2. Court-Brown CM, Caesar B. Epidemiology of adult fractures: a review. Injury 2006;37:691-7.

3. Melton LJ III, Crowson CS, O'Fallon WM. Fracture incidence in Olmsted County, Minnesota: comparison of urban with rura rates and changes in urban rates over time. Osteoporos Int 1999;9:29-37.

4. Court-Brown CM, McBirnie J, Wilson G. Adult ankle fractures-an increasing problem? Acta Orthop Scand 1998;69:43-7.

5. Martin AG. Weber B ankle fracture: an unnecessary fracture clinic burden. Injury 2004;35:805-8.

6. Clare MP. A rational approach to ankle fractures. Foot Ankle Clin 2008;13:593-610.

7. Phillips WA, Schwartz HS, Keller CS, et al. A prospective, randomized study of the management of severe ankle fractures. J Bone Joint Surg Am 1985;67:67-78.

8. Michelson JD. Fractures about the ankle. J Bone Joint Surg Am 1995;77:142-52.

9. Michelson JD, Magid D, McHale K. Clinical utility of a stability-based ankle fracture classification system. J Orthop Trauma 2007;21:307-15

10. Van Schie-Van der Weert EM, Van Lieshout EMM, De Vries MR, et al. Determinants of outcome in operatively and non-operatively treated Weber-B ankle fractures. Arch Orthop Trauma Surg 2012;132:257-63

11. Murray AM, McDonald SE, Archbold P, et al. Cost description of inpatient treatment for ankle fracture. Injury 2011;42:1226-9. 
12. SooHoo NF, Krenek L, Eagan MJ, et al. Complication rates following open reduction and internal fixation of ankle fractures. J Bone Joint Surg Am 2009;91:1042-9.

13. Slobogean GP, Marra CA, Sadatsafavi M, et al. on behalf of the Canadian Orthopedic Trauma S. Is surgical fixation for stress-positive unstable ankle fractures cost effective? Results of a multicenter randomized control trial. J Orthop Trauma 2012;26:652-8

14. Ansari U, Adie S, Harris IA, et al. Practice variation in common fracture presentations: a survey of orthopaedic surgeons. Injury 2011;42:403-7.

15. Koval KJ, Egol KA, Cheung $\mathrm{Y}$, et al. Does a positive ankle stress test indicate the need for operative treatment after lateral malleolus fracture? A preliminary report. $J$ Orthop Trauma 2007:21:449-55.

16. Egol KA, Amirtharajah M, Tejwani NC, et al. Ankle stress test for predicting the need for surgical fixation of isolated fibular fractures. J Bone Joint Surg Am 2004;86:2393-8.

17. Johanson NA, Liang MH, Daltroy L, et al. American Academy of Orthopaedic Surgeons lower limb outcomes assessment instruments. Reliability, validity, and sensitivity to change. J Bone Joint Surg Am 2004;86-A:902-9.

18. Suk M. Musculoskeletal outcomes measures and instruments. Thieme, 2009:1

19. Hunsaker FG, Cioffi DA, Amadio Peter C, et al. The American Academy of Orthopaedic Surgeons outcomes instruments: normative values from the general population. $J$ Bone Joint Surg Am 2002;84-A:208-15.

20. Ware JJ, Kosinski M, Keller SD. A 12-Item Short-Form Health Survey: construction of scales and preliminary tests of reliability and validity. Med Care 1996;34:220-33.

21. Ware JE Jr, Kosinski M, Turner-Bowker DM, et al. User's manual for the SF-12v2 health survey with a supplement documenting SF-12 health survey, Lincoln, RI: QualityMetric Incorporated, 2002.

22. Sultan MJ, Zhing T, Morris J, et al. Compression stockings in the management of fractures of the ankle: a randomised controlled trial. Bone Joint J 2014;96-B:1062-9.
23. So S, Harris IA, Naylor JM, et al. Correlation between metal allergy and treatment outcomes after ankle fracture fixation. $J$ Orthop Surg (Hong Kong) 2011;19:309-13.

24. Dindo D, Demartines N, Clavien P-A. Classification of surgical complications: a new proposal with evaluation in a cohort of 6336 patients and results of a survey. Ann Surg 2004;240:205-13.

25. Resnick B, Nahm ES. Reliability and validity testing of the revised 12-item Short-Form Health Survey in older adults. J Nurs Meas 2001;9:151-61.

26. Mittal R, Harris IA, Adie S, et al. Factors affecting patient participation in orthopaedic trials comparing surgery to non-surgical interventions. Contemp Clin Trials 2016;3:153-7.

27. Timmermans $S$, Mauck $A$. The promises and pitfalls of evidence-based medicine. Health Affairs 2005;24:18-28.

28. Pakarinen HJ, Flinkkilä TE, Ohtonen PP, et al. Syndesmotic fixation in supination-external rotation ankle fractures: a prospective randomized study. Foot Ankle Int 2011;32:1103-9.

29. Kortekangas TH, Pakarinen HJ, Savola O, et al. Syndesmotic fixation in supination-external rotation ankle fractures: a prospective randomized study. Foot Ankle Int 2014;35:988-95.

30. Bauer M, Bergstrom B, Hemborg A, et al. Malleolar fractures: nonoperative versus operative treatment. A controlled study. Clin Orthop 1985;199:17-27.

31. Makwana NK, Bhowal B, Harper WM, et al. Conservative versus operative treatment for displaced ankle fractures in patients over 55 years of age. A prospective, randomised study.[see comment]. $J$ Bone Joint Surg Br 2001;83:525-9.

32. Rowley DI, Norris SH, Duckworth T. A prospective trial comparing operative and manipulative treatment of ankle fractures. J Bone Join Surg $\mathrm{Br}$ 1986;68:610-13.

33. Sanders DW, Tieszer C, Corbett B, Society OBOTCOT. Operative versus nonoperative treatment of unstable lateral malleolar fractures: a randomized multicenter trial. J Orthop Trauma 2012;26:129-34.

34. Lin Chung-Wei C, Donkers Nicole AJ, Refshauge Kathryn M, et al. Rehabilitation for ankle fractures in adults. Cochrane Database Syst Rev 2012 [cited 2015 Oct 29]. http://onlinelibrary.wiley.com/doi/10. 1002/14651858.CD005595.pub3/abstract 